\title{
Analysis of Effects of Domestic Wireless Equipments on Living Beings
}

\author{
Vijay Kumar, Tanu Taluja, Jasmeet Kalra, Kamlesh Kukreti, Shipra Gupta
}

\begin{abstract}
The electromagnetic waves have developed the communication system today. They are around the world completely and our environment remains under the influence of radio waves radiating from mobile phone towers and cellphones. The adverse effects of e-pollution are not subject to some form of surroundings. Radioactive radiation directly affects biomolecules such as DNA and produces genetic toxicity. In addition to neuron damage, character disorder has seen in flying birds and mammals. The radiation can have an impact on the reproduction of animals and mammals and is therefore a major cause of concern in the present.
\end{abstract}

Keywords: Electro-pollution, Electromagnetic radiation, Telecommunication. Health effects

\section{INTRODUCTION}

Electromagnetic Radiations have been a boon to mankind but it has small ill effects on living beings at the same time. The e-magnetic waves have developed the communication system today. They are around the world completely and our environment remains under the influence of radio waves radiating from stations and cellphones. The adverse effects of e-pollution are not subject to some form of surroundings. Radioactive radiation directly affects bio-molecules such as DNA and produces genetic toxicity. In addition to neuron damage, character disorder has seen in flying birds and mammals. Symptoms of locomotors have been observed in the months leading up to the their inability to fly. The radiation can have an impact on the reproduction of animals and even mammals and is therefore a major cause of concern in the present Numerous specialists say that the electromagnetic field created from radiowaves and microwaves which surround us, impact human body. Few say that it impact human body and some paper recommend it can cause different kinds of malignant growth and disorders in human interior organs like mind ,kidney, lungs and progressively one side we see tremendous populace utilizing telephone or more we see they have exceptionally incredible effect on individuals yet on the chance that they truly hurting individuals, at that point it is actually a decent issue on which we should discuss . Some paper investigate proposes that the electromagnetic waves truly affecting human body and furthermore add on this likewise affecting human intellectually additionally

Revised Manuscript Received on April 25, 2019.

Vijay Kumar, Professor, Department of Physics, Graphic Era Hill University, Dehradun, India

Tanu Taluja, Student BSc, Department of Physics, Graphic Era Hill University, Dehradun, India

Jasmeet Kalra, A. P. Department of Mechanical Engineering, Graphic Era Hill University, Dehradun, India.

Kamlesh Kukreti, Department of Electronics and Communication Engineering, Graphic Era Deemed to be University, Dehradun, India

Shipra Gupta, Associate Professor, Department of Commerce, Graphic Era Hill University, Dehradun, India cell phone currently turning out to be instrument to get loosened up the normal time which the greater part of the individuals giving from that point 24 hour time to versatile is more noteworthy than the time which provide for others works and in this classification youngsters rank is number one. Now a days every teenager is requesting cell phone and it's interest is increasing and the greater part of total populace is utilizing cell phones. As per 2020 information there are roughly 4.5 cell phones clients in world from which 3.8 are cell phone clients, as indicated by 2018 information in India we have 390 million cell phone clients. As indicated by American 2019 survey- American individuals investing third quarter of their energy in cell phones $49 \%$ via web-based networking media, $36 \%$ on email and $32 \%$ percent on programs.

Some examination papers likewise recommend that kids are in peril because of cell phone radiation since they invest their energy playing internet games as children under 20 age are in incredible hazard because of radiation this radiation is affecting their mind a few analysts said the kids having their own cell phones before 20 having hazard that cerebrum tumour may happen in their cerebrum. Around 1948 it contact by 30 million individuals in USA because of phone life is changing so quickly that individuals' said that this gadget in coming time will turn out to be more natural product full for us as we probably are aware now cell phones have such a large number of features that even now individuals don't realize what are the complete number of features in phone it have- time clock, alarm ,calculator, calender, reminder.

The realized direct wellbeing impacts of radio-recurrence (RF) radiation are because of assimilation of vitality from the radio waves into the body causing heating up of tissues. Wellbeing hazards develop if the human temperature guideline can't kill the abundance heat. The wellbeing impacts because of increment in temperature show up during the introduction or following it. These scientifically demonstrated hurtful wellbeing impacts go about as a premise for the introduction furthest reaches of general open. A similar breaking point esteems are utilized, aside from a couple of special casesin all EU nations. Direct wellbeing impacts because of RF radiation would assume that the introduction cut off points of overall population are surpassed multi-overlay. RF exposures of this extent happen just in remarkable working conditions, as in pole activities, radar support what's more, modern high-recurrence warming. In typical conditions the most noteworthy introduction is brought about by gadgets kept near the body, for example, portable telephones and different remote terminal gear.

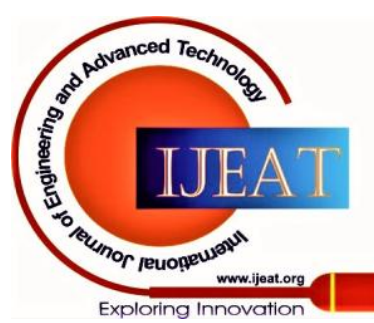




\section{Analysis of Effects of Domestic Wireless Equipments on Living Beings}

These gadgets initiate at most an introduction of about the equivalentsize as the legal introduction limits. High-power fixed transmitters, for example, cell phone base stations what's more, TV transmitters, cause a significantly littler introduction on the grounds that their reception apparatuses are introduced on high poles or rooftops where individuals have no get to. The exposures are as a rule short of what one thousandth or a few ten thousandth parts, and even at the most, just a couple of percents of the introduction limits.

A few examinations show that dosages near the cut off esteems may instigate natural responses in cells that are not clarified by the warming up of tissues. At present we don't realize enough to evaluate if these responses have any impact on wellbeing what's more, in this manner further examinations are fundamental. Cell phones are the major cause for RF radiation Cell phones are the most significant wellspring of RF fields today in our condition. The most noteworthy presentation brought about by them can be of the equivalent size as far as possible.

The introduction to RF radiation brought about by a cell phone is given as a SAR (specific assimilation rate) esteem that is reported model-specifically in the cell phone's client manual. Since there is yet no sufficient information on wellbeing impacts due to RF presentation, STUK states that it is sensible to limit kids' utilization of cell phones. A kid's life form is as yet creating and in this way more touchy to outer burdens than that of a grown-up. Too the lifetime introduction will be longer for a kid than for somebody who has begun to utilize cell phones as an adult.

A few components influence the RF presentation of a cell phone client. Most significant are transmit intensity of the cell phone and the amount of the transmitted vitality of the radio waves are caught up in the telephone client's head. The transmit power depends on the nature of the association between a cell phone and a base station; the closer the base station, the lower the transmit intensity of a cell phone. The cell phone continuously modifies the force as low as conceivable so the association with base station is as yet kept up. The client can diminish the introduction best utilizing a sans hands gadget what's more, setting the telephone for example Along these lines the vast majority of the vitality discharged by the cell phone is coordinated towards the base stations and as it were an extremely little part is consumed by the client.

\section{REVIEW OF LITERATURE:}

Electromagnetic waves radiation is discharged by man-made and characteristic sources and they have significant influence in our life. Electromagnetic (EM) waves is comprises of both electric and attractive field segments which is commonly opposite to the course of proliferation of the waves. These waves incorporates radio wave, infrared radiation, noticeable light, bright radiation, X-beam and Gamma beams. These EM waves are wherever so individual are constantly presented to these radiation. Long introduction prompts the danger of cardiovascular malady . EM radiation impacts muscles and nerve cells. Many skin related manifestations watched, for example, migraine, cardiovascular illness, consuming sensation, redness, exhaustion. The radiation produced by PCs, PC and PC segments are known as incredibly low recurrence (ELF) EM radiation. These equivalent sort of radiation are transmitted from TVs, electrical cables, and other electronic apparatus . Force of the radiation originating from the PC and workstations is less however as client is a lot nearer to the PC and PC it will produces medical issues. Radiation in which client presented to this radiation are radio waves, bright light and noticeable and incredibly low recurrence [1]. PC visual Display unit (VDU) are ordered into two kinds that is cathode beam tube (CRT) and Liquid gem show (LCD). CRT is vacuum based cylinder in which light emission is anticipated on glowing screen to see picture. CRT used to shape picture like in PC screen and oscilloscope and so forth. LCD is current based innovation that utilizations adjusting of fluid gem join with polarizer which are utilized in PC screen. Radiation from such sort of gadgets (VDUs) causes strain and dryness in eyes and disturbance. These radiation (ELF) can cause different wellbeing peril like trouble in rest and hypersensitivity additionally can cause malignant growth heart issue and Alzheimer ailments [2]. As we probably are aware CRT is utilized to location of development of baby in pregnant lady long utilization of CRT could cause High paces of irregular birth and unsuccessful labours. In EM waves attractive segments is more infiltrated than electric segments in person likewise radiation rot quickly as the separation is expanded from the source. Electromagnetic radiation is the fundamental driver of current innovative and budgetary development. Over the top utilization of EM wave based electrical gadget has some risky effects on human wellbeing[3].

This paper examinations the majority of the negative effects, for example, Brain tumours, tangible framework breakdown, lymphatic framework down, gonad and spermatogenesis problem, malformation of ligament from demonstrated investigations examination. Separate in vivo and in vitro examinations result gathered in the paper. A definitive finding is whether em wave is hazardous or not. There are two kinds of Electromagnetic radiation; ionizing radiation and non-ionizing radiation [4].

\section{EFFECTS OF ELECTROMAGNETIC WAVES:}

Remote correspondence has been utilized over the worldwide in numerous years for highlight point (p2p) and highlight multipoint network. AM and FM radio, transmission stations, portable and phones, radar and microwave frameworks are the most common remote arrangements. The electromagnetic (EM) range has scope of frequencies like Extremely Low Frequency (ELF) and Very Low Frequency (VLF), through Radio Frequency (RF) and Microwaves, to Infrared (IR) light, Visible Light, Ultraviolet (UV) light, X-beams, and Gamma beams. human heath problems due to electromagnetic radiation Rough metals powerlessness and electromagnetic affectability Mirtazapine and his scientists bunch has found Electro-extreme touchiness (EHS) from electromagnetic radiation. 
Electro-excessive touchiness (EHS) is the scattered physiological procedures related with malady or injury of EHS and it is less critical. Likewise scientists demonstrated that it is connected with huge metallic component. Strong metallic component appended with the proteins inside tissues and organs are accepted to have less peril [6].

Likewise, Mirtazapine and partners have seen that consistent attractive field as a rule created from cell phones and different remote gadgets may influence mercury fume discharge from dental amalgam. The weakened mercury angle is expanding in salivation inside amalgam transporters. Consequences for tactile framework and mental issue Cellular telephones are being utilized near mind tissue. Henceforth cerebrum tissue is affected by electromagnetic wave for the most part. Numerous investigations show that human tangible framework and conduct are influenced intently by the radio recurrence electromagnetic waves coming edge the base stations(BTS) [7] .

\section{IMPACT ON HUMANS}

There are numerous kinds of electromagnetic waves yet the wave which is helping here is radio waves and the oscillation of electrical and attractive field make radio Frequency (RF).When you make call, the radio waves created by your transmitter in your phone spread the radio waves every which way the waves can be ingest or reflected by the material present in the region of them, before coming to their closest base station [11].

Every cell phone contain a radio receiving wire which is utilized by it to impart or get radio signs, reception apparatus is that part which work is to change over electrical signs into radio waves. In people, radiofrequencies initiate natural impacts on bio atoms that remember changes for intracellular ionic focus, cell expansion, obstructions with safe framework, consequences for creature's regenerative limit, impacts on pressure hormones in intrauterine turn of events, genotoxic impacts, consequences for the anxious and circulatory framework, and a decrease in the quantity of births. Various examinations have pinpointed wireless towers as a potential reason in the decay of creature populaces. Creatures presented to electromagnetic fields can endure a weakening of wellbeing and changes in conduct [12].

Radio wire is fundamentally comprised of copper in more established mobile phone it is outside the PDA yet today innovation minimized it so much that presently it's size is so little you don't ready to the ocean where it is the past recieving wire telephone which reception apparatus is outside the gadget is hard to convey in pocket since its receiving wire may impact with the assistance of innovation we expel this trouble.

In a cross sectional case control study directed to contemplate hereditary harm in people living in region of portable base stations, it was discovered that power thickness in the region inside $300 \mathrm{~m}$ from the base station surpassed as far as possible and was altogether higher contrasted with the region from where control tests were gathered. Hereditary harm parameters of DNA movement length, harm recurrence (DF) and harm file were fundamentally raised in the example bunch contrasted with particular qualities in sound controls. Normal and long haul utilization of microwave gadgets (cell phone, microwave) at residential level can have negative effect upon natural framework particularly on mind. The expanded responsive oxygen species (ROS) assume a significant job by upgrading the impact of microwave radiations which may cause neurodegenerative illnesses [10].

The non methodical and unexpected calcium efflux can show in numerous inconsistencies of neuronal control as calcium particle assumes a significant job in the elements of the sensory system, for example, the arrival of synapses.

In addition, RFR enacts endogenous narcotics in the mind, which thusly causes a decline in cholinergic movement prompting momentary memory shortfall. The pressure hormone "corticotrophin discharging factor" is likewise included. The occupants around versatile base station receiving wires essentially gripe or create cerebral pain, memory changes, tremors, unsteadiness, and burdensome manifestations and rest unsettling influence than controls [13].

\section{ADVERSE EFFECTS ON ANIMALS}

There are numerous kinds of electromagnetic waves yet the wave which is helping here is radio waves and the oscillation of electrical and attractive field make radio Frequency (RF).When you make call, the radio waves created by your transmitter in your phone spread the radio waves every which way the waves can be ingest or reflected by the material present in the region of them, before coming to their closest base station .

In local creatures, for example, hamsters and guinea pigs, living close to portable media transmission base stations have been seen. Electromagnetic radiation can apply an aversive social reaction in bats. Exploratory investigations on rodents have uncovered cell phone introduction affects sperm motility prompting diminished ripeness in guys and dystrophic changes in regenerative organs in female rodents. Not just earthly species, creatures of land and water have similarly endured the worst part of unpredictable mushrooming of PDA towers. Creatures of land and water are significant segments of the biological system and solid bio-markers; their wet skin, liberated from drops, hair or quills, is profoundly penetrable to water synthetic concoctions (especially hatchlings) and air poisons (particularly grown-ups). These attributes make creatures of land and water particularly delicate to ecological conditions, changes of temperature, precipitation or bright (UV) radiation and dependable screens of neighbourhood conditions. Radiation transmitted by telephone poles influences the improvement of tadpoles prompting expanded mortality. Vanishing of creatures of land and water and different life forms is a piece of the worldwide biodiversity emergency which can have genuine ramifications on the natural parity. The broad utilization of cell phones has been joined by open discussion about conceivable unfriendly consequences for human wellbeing. 


\section{Analysis of Effects of Domestic Wireless Equipments on Living Beings}

In any case, little is thought about the impacts of long haul introduction creatures and different vulnerable animals occupying close to mobile phones [14].

It state that one could never observe birds, remaining close to the mobile tower, the explanation expanded assimilation of radiation inferable from huge surface region of fowl . liquid substance is little, it gets warmed up quick and furthermore the attractive field upsets their navigational

\section{ADVERSE EFFECTS OF RADIATION ON BIRDS}

The broad utilization of cell phones has been joined by open discussion about conceivable unfriendly consequences for human wellbeing. In any case, little is thought about the impacts of long haul introduction creatures and different vulnerable animals occupying close to mobile phones. It state that one could never observe birds, remaining close to the mobile tower, the explanation expanded assimilation of radiation inferable from huge surface region of fowl . liquid substance is little, it gets warmed up quick and furthermore the attractive field upsets their navigational abilities.

At the point when flying creatures are presented to frail electromagnetic fields, they perplex also, start to fly every which way, which clarify transitory winged animals sabotaging capacities. A large number of transitory winged creatures pass on every year from impacts with media transmission poles. Winged creatures are accepted to utilize earth's attractive field for route, and get seriously confused by the radiation media transmission poles. ongoing a stamped decrease of the house sparrow populace. London has witnessed a precarious fall in its sparrow populace; a 75 percent fall since 1994. There have been sensational decays, nearly to the point of eradication in Glasgow, Hamburg, Dublin, Belgium, and so forth.

Conduct changes have additionally been seen among flying creatures near the telephone receiving wires. Visit battling, youthful one mortality and relinquished homes have been seen in region of telephone reception apparatuses. In an examination, $75 \%$ of chicken incipient organisms that were presented to a GSM cell phone during hatching passed on contrasted with $16 \%$, who were not presented to any radiation. The eggs laid in homes close to towers neglect to bring forth. A general vanishing of flying creatures like Kestrel, White Stork, Rock Dove, pigeons, Magpie has been seen close to portable media transmission base stations. Train issues, rearing issues, and propensity to remain long in lower portions of the trees and on the ground have been watched [15].

\section{EFFECTS ON INSECTS}

Creepy crawlies types of biological system touchy to in SA has uncovered a solid connection between abatement in subterranean insect and creepy crawly decent variety with the electromagnetic radiation presentation.

An abatement of creepy crawlies and 8-legged creature close has been distinguished and certified by specialists and receiving wire's support staff. Homes close to the receiving wires, a nonattendance of flieshas been taken note. The abilities. radiation and moment changes in the earth. An investigation

waves influences advancement of bugs in a warm way. The vanishing of creepy crawlies have an impact on feathered creature's debilitating brought about by absence of food, particularly in a youthful winged creature's life., it was uncovered that uncovering organic product flies to cell phone radiation, raised pressure protein levels (Hsp70) which as a rule implies that phones are presented to unfavourable ecological conditions (non-warm stun). Einstein stated, "the honey bees vanishes from the earth man would have close to 4 yrs to live."

Rays contamination has brought in honey bees as CCD where honey bee can't discover to the live because of steady waves foundation commotion that appears to upset correspondence inside individual honey bees. in the range of 1985 and 2005 contrasted with a normal of 20 percent across Europe. As of late, a sharp decrease has likewise been seen in business honey bee populace in Kerala representing a genuine danger to bumble bees [5].

the most elevated thickness of portable towers. Comparable cases have been seen in different pieces of India and credited to expanding e-contamination in the earth. At the point when bumble bee provinces were uncovered with radiation, and zone were diminished and returning time of bumble expanded contrasted with comparable nonuncovered states. A few different investigations show that the high-recurrence emf of mobile modify the resounding improvement of living life forms and can cause changes in specific zones of their cerebrum [8].

\section{CONCLUSION}

After above analysis, it is concluded that high frequency electromagnetic waves are generated from mobile phones, mobile phone towers, screen of computer monitor, TV screen, microwaves, electric wiring inside the home, etc. When the above given non ionising electromagnetic radiation incident on human beings, animals, birds etc. it enter inside the body. Right now, we are living in umbrella of electromagnetic waves. EMW affect the living beings in many ways. The waves can affect on the brain of living beings. Many types of health effects like tumour, head ach, unpleasantness, etc are observed by the people. Thus in this manuscript, it is concluded that people keep away from the gadgets which are emitted high frequency electromagnetic waves.

\section{REFERENCES}

1. Mobile Phone Radiations and Its Impact on Birds, Animals and Human Beings

2. ShafiyaImtiazRafiqi , Saroj Kumar,Rajni Chaudhary, Uiase Bin Farooq4

3. and P. Kirthika

4. Markov M and Grigoriev Y (2015). Protect children from EMF.

5. Electromagnetic Biology and Medicine, 34: 251-256.

6. Behari J (2010). Biological responses of mobile phone frequency

7. exposure. Indian Journal of Experimental Biology, 48:

8. 959-981.

9. Moulder JE, Foster KR, Erdreich LS and McNamee JP (2005).

10. Mobile phones, mobile phone base stations and cancer: A

11. review. International Journal of Radiation Biology, 81:189-203.




12. Gandhi G, Kaur G and Nisar U (2015). A cross-sectional case

13. control study on genetic damage in individuals residing in

14. the vicinity of a mobile phone base station.

15. Electromagnetic Biology and Medicine, 34: 344-354.

16. Kesari KK, Siddiqui MH, Meena R, Verma HN and Kumar S

17. (2013). Cell phone radiation exposure on brain and

18. associated biological systems. Indian Journal of Experimental Biology, 51: 187-200.

19. Electromagnetic Radiation from Cell Phone Towers: A Potential Health Hazard for Birds, Bees and Humans By ChandaSiddoo-Atwal

20. Nihan Merve Sarıkaya, Bir işyerinde elektromanyetik alan ölçümü yapılması ve sonuçlarının iş sağlığı ve güvenliği açısından değerlendirilmesi, İş sağlığı ve güvenliği uzmanlık tezi, 2014

21. Tolga İnce, Elektromanyetik Kirlilik, Gazi Üniversitesi Yüksek Lisans 2000-60000 45.75 Tezi, 2007.

22. Turkish dwelling, Measurement, Vol.46 (9), pp. 3002-3009, November 2013.

23. Levent Seyfi, Ercan Yaldız, Can Nacaroğlu, Analyzing mobile phone 6. In 2018, A new revision on the limit values at frequencies from $10 \mathrm{kHz}$ to $94 \mathrm{GHz}$, as shown in Table 7, was carried out by Information and Communication Technologies Authority

24. electromagnetic radiation, Electronics World, Vol. 122(1962), pp.4043. June 2016.

25. Genç, O., Bayrak, M., and Yaldız, E. (2010). Analysis of the Effects of GSM Bands to the Electromagnetic Pollution in the RF Spectrum Progress In Electromagnetics Research, PIER 101, 17-32. of TURKEY.

26. Radiation from mobile towers affect birds: MoEF study 